\title{
Proof of concept of a multimodal intravital molecular imaging system for tumour transpathology investigation
}

\author{
Zhen Liu $^{1,2} \cdot$ Tao Cheng $^{1} \cdot$ Stephan Düwel ${ }^{1} \cdot$ Ziying Jian $^{1} \cdot$ Geoffrey J. Topping ${ }^{1} \cdot$ Katja Steiger $^{3} \cdot$ Qian Wang $^{1}$. \\ Rickmer Braren ${ }^{4}$. Sybille Reder ${ }^{1}$. Markus Mittelhäuser ${ }^{1}$. Christian Hundshammer ${ }^{1}$. Benedikt Feuerecker ${ }^{1}$. \\ Sung-Cheng Huang ${ }^{5} \cdot$ Markus Schwaiger $^{1} \cdot$ Franz Schilling $^{1} \cdot$ Sibylle I. Ziegler ${ }^{1,6} \cdot$ Kuangyu Shi $^{1,7,8}$ (i)
}

Received: 13 May 2021 / Accepted: 22 September 2021

(c) The Author(s) 2021

\begin{abstract}
Background Transpathology highlights the interpretation of the underlying physiology behind molecular imaging. However, it remains challenging due to the discrepancies between in vivo and in vitro measurements and difficulties of precise co-registration between trans-scaled images. This study aims to develop a multimodal intravital molecular imaging (MIMI) system as a tool for in vivo tumour transpathology investigation.

Methods The proposed MIMI system integrates high-resolution positron imaging, magnetic resonance imaging (MRI) and microscopic imaging on a dorsal skin window chamber on an athymic nude rat. The window chamber frame was designed to be compatible with multimodal imaging and its fiducial markers were customized for precise physical alignment among modalities. The co-registration accuracy was evaluated based on phantoms with thin catheters. For proof of concept, tumour models of the human colorectal adenocarcinoma cell line HT-29 were imaged. The tissue within the window chamber was sectioned, fixed and haematoxylin-eosin (HE) stained for comparison with multimodal in vivo imaging.

Results The final MIMI system had a maximum field of view (FOV) of $18 \mathrm{~mm} \times 18 \mathrm{~mm}$. Using the fiducial markers and the tubing phantom, the co-registration errors are $0.18 \pm 0.27 \mathrm{~mm}$ between MRI and positron imaging, $0.19 \pm 0.22 \mathrm{~mm}$ between positron imaging and microscopic imaging and $0.15 \pm 0.27 \mathrm{~mm}$ between MRI and microscopic imaging. A pilot test demonstrated that the MIMI system provides an integrative visualization of the tumour anatomy, vasculatures and metabolism of the in vivo tumour microenvironment, which was consistent with ex vivo pathology.

Conclusions The established multimodal intravital imaging system provided a co-registered in vivo platform for trans-scale and transparent investigation of the underlying pathology behind imaging, which has the potential to enhance the translation of molecular imaging.
\end{abstract}

Keywords Intravital imaging · Multimodal imaging · Window chamber · Tumour microenvironment · Transpathology · Positron imaging $\cdot$ Fiducial marker $\cdot$ Glycolysis imaging

This article is part of the Topical Collection on Preclinical Imaging.

Kuangyu Shi

k.shi@tum.de

1 Department of Nuclear Medicine, School of Medicine, Technische Universität München, Munich, Germany

2 Institute of Biomedical Engineering, Shenzhen Bay Laboratory, Shenzhen, China

3 Department of Pathology, School of Medicine, Technische Universität München, Munich, Germany

4 Department of Radiology, Technische Universität München, Munich, Germany
5 Department of Molecular and Medical Pharmacology, David Geffen School of Medicine, University of California, Los Angeles, USA

6 Department of Nuclear Medicine, University Hospital LMU Munich, Munich, Germany

7 Department of Nuclear Medicine, University of Bern, Bern, Switzerland

8 Department of Informatics, Technische Universität München, Munich, Germany 


\begin{tabular}{|c|c|}
\hline \multicolumn{2}{|l|}{ Abbreviations } \\
\hline$\left[{ }^{18} \mathrm{~F}\right] \mathrm{FDG}$ & 2-deoxy-2- $\left({ }^{18} \mathrm{~F}\right)$ fluoro-D-glucose \\
\hline AFN & Atipamezole/flumazenil/naloxone \\
\hline BLI & Bioluminescence imaging \\
\hline CCD & Charge-coupled device \\
\hline DCE-MRI & $\begin{array}{l}\text { Dynamic contrast-enhanced magnetic } \\
\text { resonance imaging }\end{array}$ \\
\hline FITC-dextran & Fluorescein isothiocyanate-dextran \\
\hline Gd-DTPA & $\begin{array}{l}\text { Gadolinium-diethylenetriamine penta- } \\
\text { acetic acid }\end{array}$ \\
\hline GFP & Green fluorescent protein \\
\hline$H \& E$ & Haematoxylin-eosin \\
\hline MIMI & Multimodal intravital molecular imaging \\
\hline MMF & Midazolam/medetomidine/fentanyl \\
\hline MR & Magnetic resonance \\
\hline MRI & Magnetic resonance imaging \\
\hline NEX & Number of excitation \\
\hline NIR & Near infrared \\
\hline PD & Pharmacodynamics \\
\hline PEEK & Polyetheretherketone \\
\hline PET & Positron emission tomography \\
\hline p.i. & Post-injection \\
\hline PK & Pharmacokinetics \\
\hline RF & Radio frequency \\
\hline s.c. & Subcutaneously \\
\hline TRICKS & $\begin{array}{l}\text { Time-resolved imaging of contrast } \\
\text { kinetics }\end{array}$ \\
\hline
\end{tabular}

\section{Introduction}

Transpathology is a new theory to summarize the endeavour of molecular imaging for deciphering underlying pathophysiology [1, 2], which consists of trans-scale, transparent and translational investigation of imaging signals (Fig. 1A). It holds the great potential to transparentize tissue and better present the underlying pathophysiological information, as well as to better facilitate the translational processes from the bench to the bedside. However, the practice of transpathology is complicated by discrepancies between in vivo imaging and ex vivo pathology. Conventionally, tissues need to be sampled or resected for the investigation by microscopy [3-6]. Although these in vitro methods have been widely used in various applications, they are destructive and have limited ability to provide insight into in vivo dynamics [7]. Another crucial problem for the comparison between imaging and pathology is the co-registration of in vivo and ex vivo measurements. It is difficult to find useful landmarks or similarities between the images with such a huge resolution difference and typical co-registration algorithms are not applicable [8]. Also, the complicated preparation procedure of the histology sections introduces substantial distortions, and it is thus almost impossible to localize the physiological features precisely in conventional imaging methods.

Intravital imaging is a powerful tissue investigation method to provide high-resolution information of the tissue microenvironment in an intact animal [9, 10]. Different transparent window models have been developed to investigate various types of tumours. Chronic window models such as dorsal skin windows and cranial windows expose a stable tumour microenvironment and allow longitudinal
Fig. 1 A The concept of transpathology research and its challenges; B a concept of integrative MIMI system based on an animal model with a dorsal skin window chamber for transpathology investigation, which enables positron imaging, MRI and optical imaging within the same intact framework to allow better co-registration; $\mathbf{C}$ a detailed sketch of the designed dorsal skin window chamber with mounting pins to fix a positron camera and optical coordinate system, fixation screw threads and suture holes as further fiducial markers for localization with imaging such as MRI
A

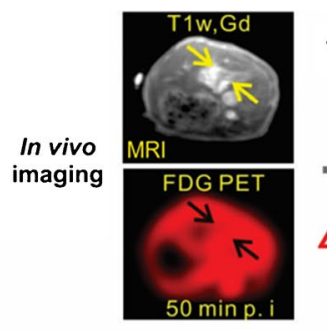

Transpathology

- Trans-scale

- Transparent

- Translation

- Discrepency: in vivo vs ex vivo

- Difficulty: co-registration
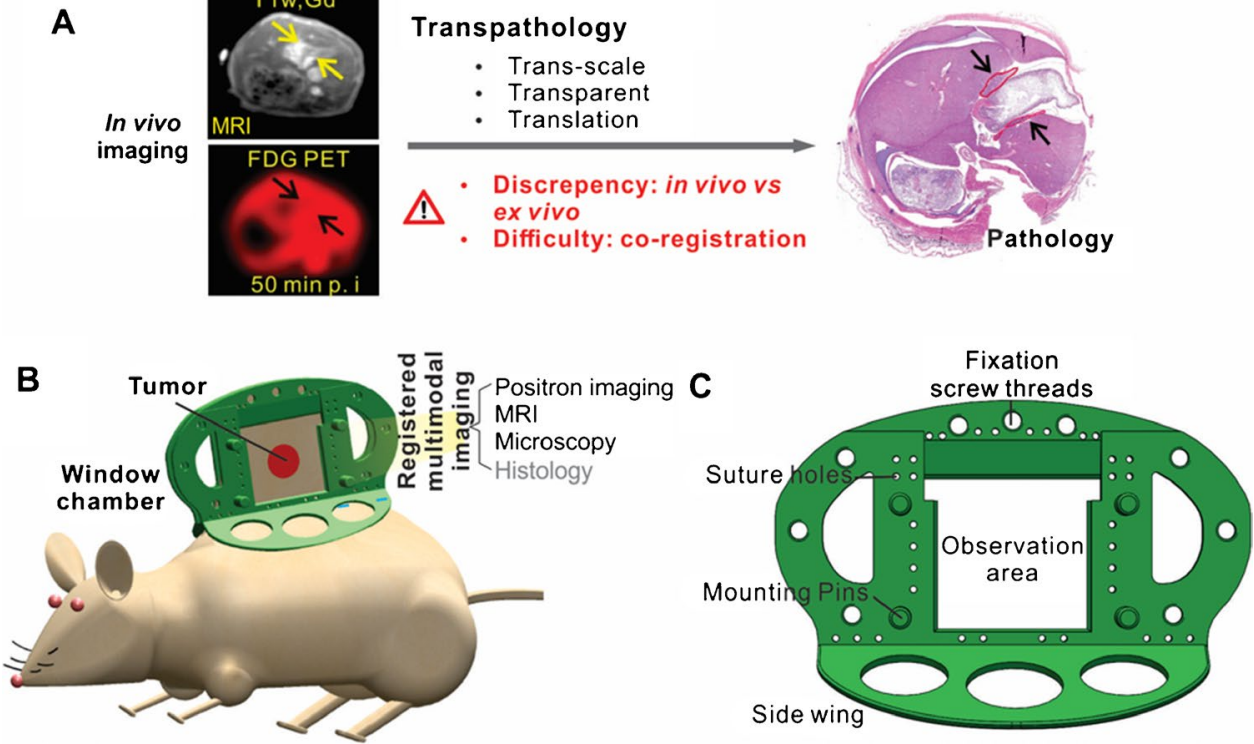
observation of tumour evolution [11, 12]. Intravital imaging can identify in vivo tissue morphological and metabolic characteristics like $\mathrm{pH}$ or $p \mathrm{O}_{2}$, using various investigation methods such as fluorescence microscopy [13-15] and phosphorescence lifetime imaging $[16,17]$. The integration of clinical imaging such as MRI or PET with intravital imaging facilitates the identification of detailed physiological features of imaging elements directly from the same intact tissue $[18,19]$. In that study, a dedicated surface receiver coil was applied to obtain high-quality MRI [18]. With careful design of the dynamic protocol, DCE-MRI was acquired to quantify the blood perfusion of the tissue [18]. In another study, direct positron imaging was proposed to improve the sensitivity and resolution of PET imaging within a window chamber [19].

The aim of this study is to establish a multimodal intravital molecular imaging (MIMI) system to support the investigation of transpathology (Fig. 1B). It is based on animal model with a dorsal skin window chamber, which is compatible with the high-resolution MRI, positron imaging and microscopic imaging and which allows precise co-registrations. A pilot test of the multimodal trans-scale imaging and the co-registration accuracy was performed.

\section{Materials and methods}

\section{Dorsal skin window chamber for multimodal intravital imaging}

For transpathology research, a MIMI system is proposed to integrate positron imaging, MRI and optical imaging (e.g. microscope) within an intact and rigid frame (Fig. 1B). This rigid frame (Fig. 1C) is established using a dorsal skin window chamber, which is compatible with the abovementioned imaging modalities, including fiducial markers for positioning and localization. The chamber is made from polyetheretherketone (PEEK), which is rigid but flexible, and is chemically inert to most organic and inorganic chemicals and solvents [18]. The mounting pins can be used to fix a positron camera accurately within the chamber. The fixation screw threads and suture holes provide additional fiducial markers for localization with imaging such as MRI, fluorescence imaging and positron imaging.

\section{Window chamber implantation and tumour transplantation}

Athymic RNU nude rats (Crl: NIH-Foxn1rnu, Charles River) were employed for the in vivo test of the MIMI system. The implantation of the multimodal compatible dorsal skin window chamber onto the RNU rat followed the literature [20,21] with some adaptions. During the chamber implantation, rats were anaesthetized with inhalation of isoflurane ( $2 \%$ in oxygen, $2 \mathrm{~L} / \mathrm{min}$ ). A single-cell suspension of the human colorectal adenocarcinoma cell line HT29 $\left(2 \times 10^{6}\right.$ cells in $0.05 \mathrm{ml}$ PBS $)$ was prepared for subcutaneous injection onto the fascia side of the intact skin layer within the window area. All animal studies were approved by the local government committee for animal protection and welfare (Tierschutzbehörde, Regierung von Oberbayern, with licence protocol number 18-13).

\section{Positron imaging}

A positron camera with a physical resolution of $230 \mu \mathrm{m}$ was employed for high-resolution 2D imaging within the window chamber area [22, 23]. The window chamber with intact skin side was fixed to an adapter (Supplemental Fig. 2), and the glass side was then mounted to the positron camera via the above-mentioned mounting pins (Supplemental Fig. 4). The cover glass was removed before mounting to the positron camera. A Mylar sheet $(6 \mu \mathrm{m})$ was placed on the surface of the camera to prevent possible contact with tissue fluid. Positron imaging of $10 \mathrm{~min}$ was acquired at $50 \mathrm{~min}$ post-injection of ca. $150 \mathrm{kBq} / \mathrm{g}$ body weight of $\left[{ }^{18} \mathrm{~F}\right] \mathrm{FDG}$ under anaesthesia.

\section{MR imaging}

The MR images were scanned with a $7 \mathrm{~T}$ preclinical MRI system (Discovery MR901, Agilent Technologies, Santa Clara, USA). The rat was fixed on the MR bed, with air heating during anaesthesia and monitoring of the heart rate by electrocardiogram, breathing rate with a pressure-sensitive pad (RAPID Biomedical $\mathrm{GmbH}$ ), and body temperature. A single-channel flexible radiofrequency (RF) receiver coil (diameter $30 \mathrm{~mm}$, RAPID Biomedical $\mathrm{GmbH}$ ) was placed on top of the window chamber. A quadrature birdcage resonator (72-mm inner diameter, RAPID Biomedical $\mathrm{GmbH}$ ) was used for RF transmission. T1-weighted images using a gradient recalled echo sequence $(\mathrm{TR}=300 \mathrm{~ms}$, $\mathrm{TE}=1.1 \mathrm{~ms}, 192 \times 192$ matrix, $1 \mathrm{~mm}$ slice thickness, $4 \mathrm{~cm} \times 4 \mathrm{~cm}$ FOV, number of excitations $(\mathrm{NEX})=4$, flip angle $60^{\circ}$ ) and $\mathrm{T} 2$-weighted images using a fast spin-echo sequence $(\mathrm{TR}=3000 \mathrm{~ms}, \mathrm{TE}=32.7 \mathrm{~ms}, 192 \times 192$ matrix, $1 \mathrm{~mm}$ slice thickness, $4 \mathrm{~cm} \times 4 \mathrm{~cm}$ FOV, NEX $=4$, flip angle $90^{\circ}$ ) were acquired after positioning. Dynamic contrastenhanced (DCE) MRI was acquired during the injection of Gd-DTPA solution $(0.25 \mathrm{mmol} / \mathrm{kg})$ with a flow rate of $160 \mathrm{ml} / \mathrm{h}$ using a syringe pump (Pump Elite 11, Harvard Apparatus, Cambridge, USA). The DCE-MRI was acquired using the TRICKS sequence (time-resolved imaging of 
contrast kinetics) [24]. TRICKS acquisition was started $15 \mathrm{~s}$ before the start of the pump injection. The parameters were $\mathrm{TR}=4 \mathrm{~ms}$, $\mathrm{TE}=1.7 \mathrm{~ms}, 192 \times 192$ matrix, $1 \mathrm{~mm}$ slice thickness, $4 \mathrm{~cm} \times 4 \mathrm{~cm}$ FOV, flip angle $40^{\circ}$. Fifty-six phases were obtained with 28 images in each phase. Each phase measured with a temporal resolution of $3 \mathrm{~s}$. After the DCEMRI, another T1-weighted MRI scan was acquired with the same the parameters as before the contrast agent injection.

\section{Optical imaging}

Pathophysiological information was observed with a fluorescence microscope (Imager. M2, Zeiss, Germany) equipped with a black/white charge-coupled device (CCD) camera (AxioCam MRm). Fluorescein isothiocyanate (FITC)dextran (Sigma Aldrich, St. Louis, MO, USA) was administrated to measure perfusion under MMF (midazolam/ medetomidine/fentanyl with a concentration of $5 / 1 / 0.05 \mathrm{mg} /$ $\mathrm{ml}$ ) anaesthesia. The window chamber was fixed onto the adapter (Supplemental Fig. 2) and then fixed to the fluorescence microscope. A transparent coordinate plate (Supplemental Fig. 3) was mounted onto the window chamber for localization. A fluorescence image of $1.25 \times$ magnification with a green fluorescent protein (GFP) filter was recorded.

\section{Phantom test}

To assess the co-registration accuracy for different imaging modalities, phantoms were prepared with two or three thin catheters (I.D.: $0.3 \mathrm{~mm}$, O.D.: $0.6 \mathrm{~mm}$ ) fixed independently within the window chamber using glue and transparent tape. Phantoms were imaged with positron imaging, MRI and fluorescence microscopy. The catheters were filled with $\left[{ }^{18} \mathrm{~F}\right] \mathrm{FDG}(1 \mathrm{MBq} / \mathrm{ml})$ for positron imaging, saline during
T1-weighted MRI imaging and FITC-dextran during fluorescence imaging.

\section{Ex vivo histological staining}

After imaging, the rat was euthanized by administration of $0.5 \mathrm{ml}$ pentobarbital sodium via tail vein injection under anaesthesia (Narcoren, $16 \mathrm{~g} / 100 \mathrm{ml}$, Merial GmbH, Germany). The skin along the window area was cut and fixed with $4 \%$ para-formalin solution for 1 day, then sunk into $70 \%$ ethanol for 2 days. The fixed tissue block was embedded in paraffin and cut for haematoxylin-eosin (H\&E) staining. The whole stained tissue slide was recorded by transmission light using a fluorescence microscopy (BZ-9000, KEYENCE). Due to the limited FOV of the microscopy, the tissue within the window area cannot be covered within one microscopic image. Therefore, the tissue slide was recorded one by one using a $4 \times$ objective lens. The images were further combined into a new image with a larger FOV.

\section{Data processing}

All the primary data from different imaging modalities were aligned via the fiducial markers using PMOD (PMOD Technologies Ltd., Zürich, Switzerland). For phantom measurements, the skeletons of the catheters were extracted by estimating the ridgelines of the imaging signals of the catheters with Gaussian function as medial function [25]. The errors of the co-registrations (i.e. the distances between the skeleton lines obtained from the corresponding imaging modalities) were estimated by comparing the skeleton lines.

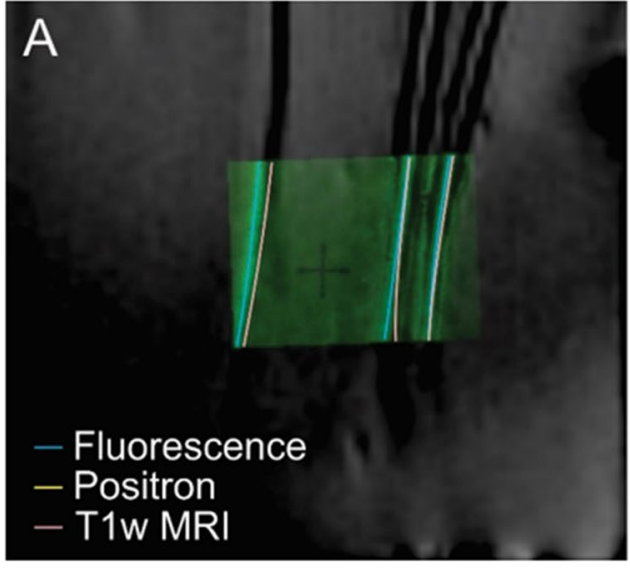

Fig. 2 An exemplary fused image of MRI, positron and fluorescence imaging of a phantom with three catheters. The catheters were first filled with $\left[{ }^{18} \mathrm{~F}\right] \mathrm{FDG}$ for positron imaging, then filled with FITC-dextran for fluorescence imaging and finally filled with saline for MRI

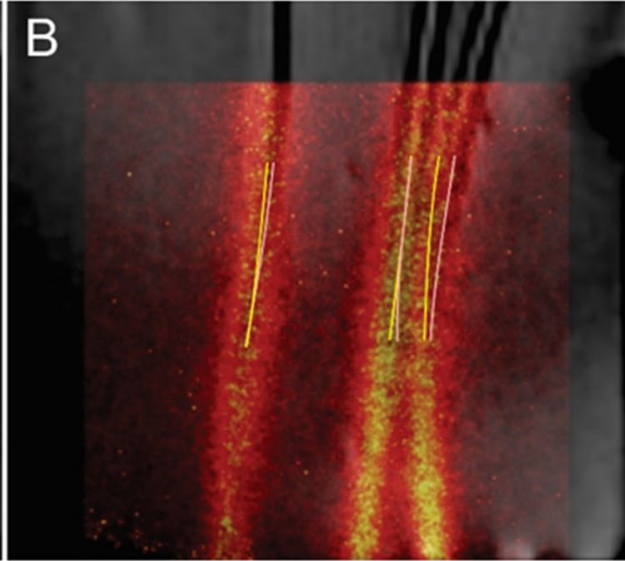

imaging. A fusion of fluorescence imaging with T1w MRI; B fusion of positron imaging with T1w MRI. The lines of different colours denote the skeletons of signals of the catheters in the corresponding imaging modalities 


\section{Results}

\section{Accuracy of image co-registration from phantom test}

Images from MRI, positron imaging and fluorescence imaging were aligned via the fiducial markers. Example phantom images are displayed in Fig. 2, with skeleton lines of different modalities extracted from each phantom tube presented on the MRI image. The registration errors between three imaging modalities are plotted in Fig. 3, with statistics for 7 skeleton lines (from three phantoms and the corresponding multimodal imaging experiments) presented in each comparison plot. The co-registration errors were $0.18 \pm 0.27 \mathrm{~mm}$ between MRI and positron imaging, $0.19 \pm 0.22 \mathrm{~mm}$ between positron imaging and microscopic imaging and $0.15 \pm 0.27 \mathrm{~mm}$ between MRI and microscopic imaging.

\section{Integrated tumour microenvironment imaging}

Example results of multimodal imaging of a rat with the window chamber are depicted in Fig. 4. The tumour (6.1 $\mathrm{mm} \times 4.9 \mathrm{~mm}$ in length and width) in the pre-contrast injection T1-weighted image was brighter compared with the surrounding tissue (Fig. 4A) and darker in the T2-weighted image (Fig. 4B). The T1-weighted MRI image after Gd-DTPA injection depicted the enhanced contrast of the tissue within the window chamber (Fig. 4C). The border of the tumour was clearer after the contrast agent injection. The $\mathrm{K}^{\text {trans }}$ map of the MR image denoting the blood flow and vascular permeability (influx rate for the contrast agent into the extravascular extracellular space tissue from blood) is presented in Fig. 4D. A fluorescence image (FITC-dextran, Fig. 4E) of the window chamber visualizes the blood perfusion around the tumour tissue. The FITC-dextran fluorescence image of a smaller FOV inside the tumour showed a detailed blood vessel network in the micrometre resolution range. The complexity of the tumour vascular network shown in the fluorescence image was consistent with the MRI observation. From the positron imaging (Fig. 4F), the tumour had generally positive $\left[{ }^{18} \mathrm{~F}\right]$ FDG uptake. From the H\&E staining image (Fig. 4G), two tumour parts were visualized. A small part of the tumour, indicated with a blue circle in Fig. 4G, was negative in positron imaging.

These three imaging modalities have trans-scaled imaging resolution. The spatial resolution of the MRI, fluorescence image and positron imaging was $156 \mu \mathrm{m}$ (pixel size), $5.16 \mu \mathrm{m}$ and $230 \mu \mathrm{m}$, respectively. The precise co-registration among these images was accomplished with the aid of the transparent plastic plate with reference pattern (refer to supplemental Fig. 3). Both the fluorescence and positron images can be precisely localized within the MRI image with the aid of the fiducial markers on the window chamber (red box in Fig. 4B). The location of the positron image is always fixed with reference to the four fixation pins, as the positron camera was mounted onto the window chamber with four holes around its detector.

\section{Discussion}

\section{Co-registration between in vivo and ex vivo imaging}

Pathology is considered the gold standard for disease diagnosis. However, it is a sample and test process, facing
A MRI vs. positron imaging

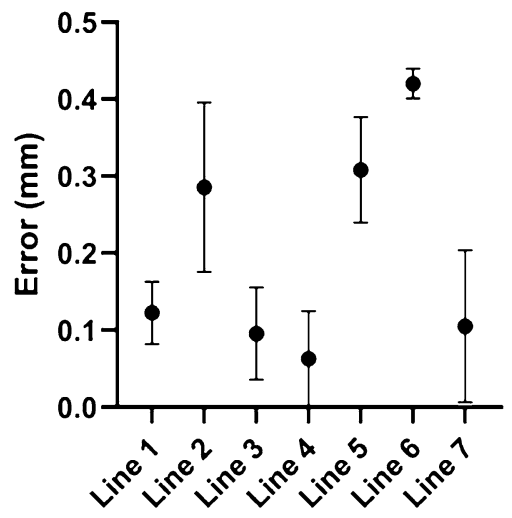

B Positron vs. microscopic imaging

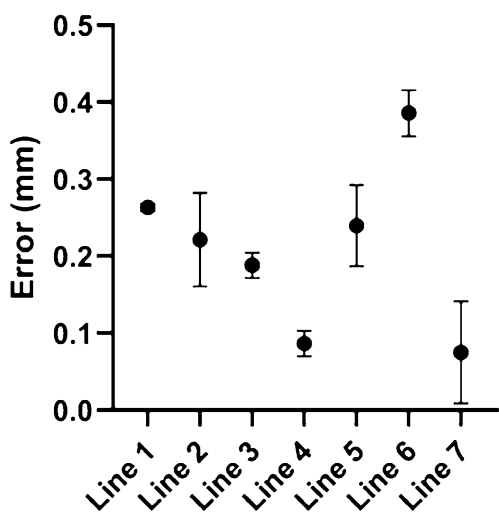

C MRI vs. microscopic imaging

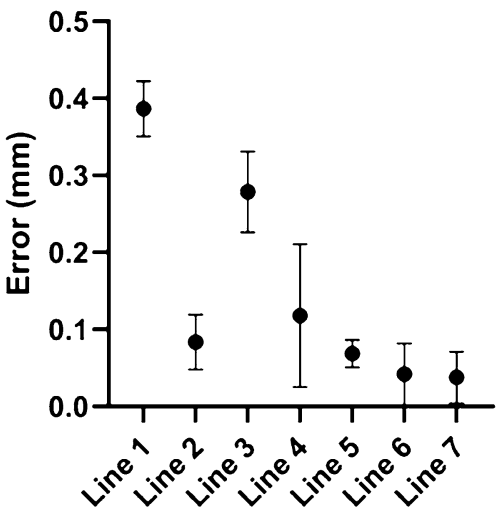

Fig. 3 The co-registration position error between the three imaging modalities of all imaged catheters (skeleton lines 1-7). The absolute distance between pairs of points along the skeleton lines is plotted for each line, and the mean and SD are plotted. A MRI and positron images; B positron and microscopy images; C MRI and microscopy images 


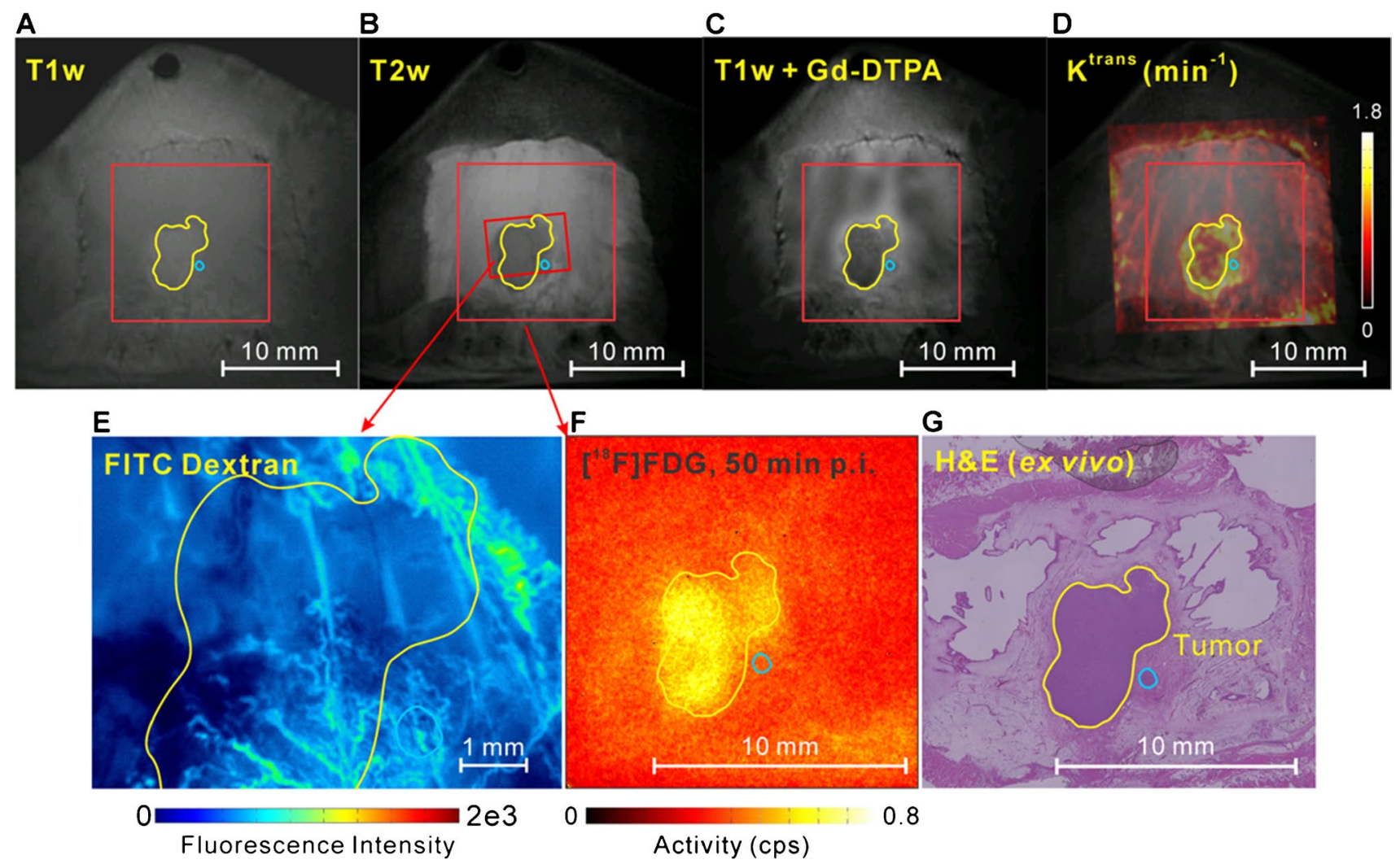

Fig. 4 Example imaging results of the multimodal intravital molecular imaging system: A a T1-weighted MR image, B a T2-weighted MR image, C a T1-weighted MR image after Gd-DTPA injection, $\mathbf{D}$ the $\mathrm{K}^{\text {trans }}$ map of the MR image; $\mathbf{E}$ a FITC-Dextran fluorescence image, $\mathbf{F}$ an $\left[{ }^{18} \mathrm{~F}\right] \mathrm{FDG}$ positron image and $\mathbf{G}$ an $\mathrm{H} \& \mathrm{E}$ staining slice of the tumour and periphery tissue. For $\mathbf{E}$ and $\mathbf{F}$, the locations of the

several limitations, such as invasiveness, incomprehensiveness due to biopsy sampling sites and sampling time, and missing in vivo changes due to limited sampling times. Imaging is a non-invasive tool for intravital observation of pathological changes. The concept of transpathology to linking the imaging with pathology faces two major obstacles for precise evaluation: (1) The discrepancies of scale and resolution between in vivo imaging and ex vivo pathology and (2) the precise co-registration among various trans-scaled imaging modalities and ex vivo pathology. Puri et al. proposed a method for accurate registration of PET images and histopathology slices [8]. They used sea urchin spines as fiducial markers for pathology and ex vivo CT imaging to localize the histopathology slices. The registration error between histopathology and ex vivo CT was reported as $0.86 \pm 0.41 \mathrm{~mm}$. The ex vivo $\mathrm{CT}$ images were then aligned to the in vivo PET images and the overall registration error between PET and histopathology was $3.0 \pm 0.7 \mathrm{~mm}$. In contrast to the registration via ex vivo anatomical imaging, the MIMI system employs images were delineated with the red boxes in $\mathbf{B}$ and linked with red arrows. Yellow and blue outlines depict the tumour locations in the tissue initially from histology images and then transferred to other measurements. Note that the H\&E image was manually co-registered to the in vivo images without the support fiducial markers

a window chamber and assisting holders to immobilize the tissues during the imaging of different modalities. Fiducial markers were designed for the physical localization of the positron imaging and MRI. The localization of microscopic imaging is more difficult due to large differences in the field of view (FOV) and resolution. A transparent plastic reference mounted to the fiducial markers with oriented coordination assists the alignment of the microscopic images. The microscopic imaging is very sensitive to the tilting of the object. The special holder of the window chamber during microscopic imaging ensured that the window chamber was placed parallel to the microscopy's lens to avoid imaging blurring. These special designs of the MIMI system led to much smaller co-registration errors of $0.18 \pm 0.27 \mathrm{~mm}$ between MRI and positron imaging, $0.19 \pm 0.22 \mathrm{~mm}$ between positron imaging and microscopic imaging and $0.15 \pm 0.27 \mathrm{~mm}$ between MRI and microscopic imaging. The immobilized imaging and the fiducial coordination of the MIMI system facilitate accurate trans-scale image co-registration. 


\section{Multimodal molecular imaging}

The combination of PET and MRI provides multimodal clinical or preclinical imaging to visualize anatomical, functional and metabolic features to support the optimization of diagnosis and therapy [26-29]. However, the increased complexity of overlapping or contradictory signal between different modalities within the same tumour due to intratumoural heterogeneity poses substantial challenges to the investigation of underlying physiology $[30,31]$. The integration of PET, MRI and intravital imaging in the MIMI system allows fingerprinting the physiological heterogeneity behind multimodal imaging for the same intact tissue $[18,19]$ and is therefore complementary to conventional preclinical PET/ $\mathrm{MR}$ in the investigation. The quasi-2D imaging on the intravital window allows higher resolution than conventional 3D PET or MR imaging. With surface coils, it is possible to focus on the thin skin tissue for imaging with a relatively high sensitivity and resolution $(<200 \mu \mathrm{m})$ [18]. For the imaging of PET tracers, conventional 3D preclinical scanners are not optimal for imaging of thin tissue within the observation window due to low intrinsic resolution and partial volume effects (PVE) [32]. The positron camera has an intrinsically higher resolution of $230 \mu \mathrm{m}$ for $2 \mathrm{D}$ imaging [ 22 , 23], which is better than the intrinsic resolution of typical 3D preclinical PET scanners [33, 34, 35]. In practice, positron imaging is not perfect $2 \mathrm{D}$ imaging either and measures the superposed signals from tissues close to the contacting surface. The positron imaging has only spill-in from tissues below the surface, while conventional 3D preclinical PET can have both spill-out and spill-in to the imaging surface. The sensitivity of the positron camera is depth dependent. The spill-in decreases as the depth increases, with half at a depth of $235 \mu \mathrm{m}$ and vanishes at a depth of $470 \mu \mathrm{m}$ [36]. In contrast, the spill-in for a high-resolution 3D preclinical PET with $1 \mathrm{~mm}$ intrinsic resolution can be $80 \%$ from tissue at a depth of $235 \mu \mathrm{m}$ and $50 \%$ at a depth of $470 \mu \mathrm{m}$, according to theoretical estimation $[37,38]$. Therefore, the proposed positron imaging is more appropriate for quasi-2D imaging of the window chamber.

\section{Limitations of the MIMI system}

The proposed MIMI system has some limitations. (1) Although the fiducial marker facilitated accuracy coregistration for the $2 \mathrm{D}$ area within the observation window, it cannot localize the depth of the microscopic image precisely (the $\mathrm{Z}$-axis that perpendicular to the $2 \mathrm{D}$ window area). (2) The registration of the ex vivo histological images and in vivo MR images of the MIMI system is still not perfect. We fixed the tissues within the window chamber with PFA initially together with the chamber during the preparation of the histology. However, some distortions and deformations happened during the demounting of the window chamber frames and during the cutting of the tissues, leading to difficulties in preserving the positions of fiducial markers. Therefore, the co-registration of ex vivo histological images and in vivo MR images still needs manual corrections in practice. (3) We used a small magnification of the microscopy in order to cover a large FOV of the tumour to allow the investigation of co-registration to in vivo imaging in this study. However, the large FOV introduced more spill-in artefacts of fluorescence imaging due to increased depth of field. (4) The current study demonstrated proof of concept of the multimodal intravital imaging system; no in-depth physiological investigation has been performed. The partial discrepancy between H\&E and positron imaging was not investigated. (5) Although the animals were treated with anti-inflammatory medication after the surgery, unspecific inflammatory $\left[{ }^{18} \mathrm{~F}\right] \mathrm{FDG}$ uptake cannot be excluded [39, 40]. (6) Here, we made a quantitative assessment about the co-registration accuracy to compare imaging modalities. However, there are other factors that might impair correlation, such as field inhomogeneities in MRI, and this was not evaluated in the study. (7) Although the MIMI system has potential for longitudinal imaging, we did not yet verify this potential.

\section{Conclusions}

A MIMI system was developed for tumour transpathology. The system provides an imaging tool combining three imaging modalities (positron imaging, MRI, fluorescence imaging). The accurate co-registration of the images obtained from the multi-scale imaging modalities ensures a comprehensive view of the tumour microenvironment. This system can bridge the discrepancies between macroscopic and microscopic images and between in vivo and in vitro images and provides a tool for the regional investigation and longitudinal observation of the underlying physiology within intact tumour tissues.

Supplementary Information The online version contains supplementary material available at https://doi.org/10.1007/s00259-021-05574-y.

Acknowledgements The authors thank Dr. Florian Gärtner, Dr. AnneKathrin v. Thaden, Dr. Sabine Schwamberger, Susanne Swirczek and Miriam Mohring for taking care of the animals and helping animal experiments. The authors thank Dr. Christine Bayer, Birgit Blechert and Dr. Daniela Schilling for providing cell lines; Prof. Markus Essler for helpful discussions and Dr. Jan Tous (CRYTUR, spol. s r.o.) for help related to the positron camera.

Funding The research leading to these results is based on the funding from German Research Foundation (DFG) Collaborative Research 
Centre 824 (SFB824) and supported by China Scholarship Council. The scientific collaboration between UCLA and TUM was supported by BaCaTec (Project 17, 2012-1).

Data availability The datasets used and/or analyzed during the current study are available from the corresponding author on reasonable request.

\section{Declarations}

Ethics approval and consent to participate All the animal studies were approved by the local government committee for animal protection and welfare (Tierschutzbehörde, Regierung von Oberbayern, with licence protocol number 18-13).

Open Access This article is licensed under a Creative Commons Attribution 4.0 International License, which permits use, sharing, adaptation, distribution and reproduction in any medium or format, as long as you give appropriate credit to the original author(s) and the source, provide a link to the Creative Commons licence, and indicate if changes were made. The images or other third party material in this article are included in the article's Creative Commons licence, unless indicated otherwise in a credit line to the material. If material is not included in the article's Creative Commons licence and your intended use is not permitted by statutory regulation or exceeds the permitted use, you will need to obtain permission directly from the copyright holder. To view a copy of this licence, visit http://creativecommons.org/licenses/by/4.0/.

\section{References}

1. Tian M, He X, Jin C, He X, Wu S, Zhou R, et al. Transpathology: molecular imaging-based pathology. Eur J Nucl Med Mol Imaging. 2021. https://doi.org/10.1007/s00259-021-05234-1.

2. Phelps M, Schwaiger M, Chiti A. Multi-scale imaging as an essential tool for precision medicine. Eur J Nucl Med Mol Imaging. 2021. https://doi.org/10.1007/s00259-021-05367-3.

3. Cho H, Ackerstaff E, Carlin S, Lupu ME, Wang Y, Rizwan A, et al. Noninvasive multimodality imaging of the tumor microenvironment: registered dynamic magnetic resonance imaging and positron emission tomography studies of a preclinical tumor model of tumor hypoxia. Neoplasia. 2009;11:247-59.

4. Bali MA, Metens T, Denolin V, Delhaye M, Demetter P, Closset $\mathrm{J}$, et al. Tumoral and nontumoral pancreas: correlation between quantitative dynamic contrast-enhanced MR imaging and histopathologic parameters. Radiology. 2011;261:456-66. https://doi. org/10.1148/radiol.11103515.

5. Hu S, Balakrishnan A, Bok RA, Anderton B, Larson PE, Nelson SJ, et al. 13C-pyruvate imaging reveals alterations in glycolysis that precede c-Myc-induced tumor formation and regression. Cell Metab. 2011;14:131-42. https://doi.org/10.1016/j.cmet.2011.04. 012 .

6. Viel T, Talasila KM, Monfared P, Wang J, Jikeli JF, Waerzeggers $\mathrm{Y}$, et al. Analysis of the growth dynamics of angiogenesis-dependent and -independent experimental glioblastomas by multimodal small-animal PET and MRI. J Nucl Med. 2012;53:1135-45. https://doi.org/10.2967/jnumed.111.101659.

7. Jain RK. The next frontier of molecular medicine: delivery of therapeutics. Nat Med. 1998;4:655-7.

8. Puri T, Chalkidou A, Henley-Smith R, Roy A, Barber PR, Guerrero-Urbano T, et al. A method for accurate spatial registration of PET images and histopathology slices. EJNMMI Res. 2015;5:64. https://doi.org/10.1186/s13550-015-0138-7.
9. Jain RK, Munn LL, Fukumura D. Transparent window models and intravital microscopy: imaging gene expression, physiological function and therapeutic effects in tumors. In: Teicher BA, editor. Tumor Models in Cancer Research: Springer; 2011.

10. Pittet MJ, Weissleder R. Intravital imaging. Cell. 2011;147:98391. https://doi.org/10.1016/j.cell.2011.11.004.

11. Oishi H, Sunamura M, Egawa S, Motoi F, Unno M, Furukawa $\mathrm{T}$, et al. Blockade of delta-like ligand 4 signaling inhibits both growth and angiogenesis of pancreatic cancer. Pancreas. 2010;39:897-903. https://doi.org/10.1097/MPA.0b013e3181 ce7185.

12. Chen X, Leischner U, Rochefort NL, Nelken I, Konnerth A. Functional mapping of single spines in cortical neurons in vivo. Nature. 2011;475:501-5. https://doi.org/10.1038/nature10193.

13. Jain RK, Munn LL, Fukumura D. Dissecting tumour pathophysiology using intravital microscopy. Nat Rev Cancer. 2002;2:26676. https://doi.org/10.1038/nrc778.

14. Vajkoczy P, Ullrich A, Menger MD. Intravital fluorescence videomicroscopy to study tumor angiogenesis and microcirculation. Neoplasia. 2000;2:53-61. https://doi.org/10.1038/sj.neo.7900062.

15. Guba M, von Breitenbuch P, Steinbauer M, Koehl G, Flegel S, Hornung M, et al. Rapamycin inhibits primary and metastatic tumor growth by antiangiogenesis: involvement of vascular endothelial growth factor. Nat Med. 2002;8:128-35. https://doi. org/10.1038/nm0202-128.

16. Dewhirst MW, Ong ET, Braun RD, Smith B, Klitzman B, Evans $\mathrm{SM}$, et al. Quantification of longitudinal tissue $\mathrm{pO} 2$ gradients in window chamber tumours: impact on tumour hypoxia. Brit J Cancer. 1999;79:1717-22.

17. Cardenas-Navia LI, Mace D, Richardson RA, Wilson DF, Shan $\mathrm{S}$, Dewhirst MW. The pervasive presence of fluctuating oxygenation in tumors. Can Res. 2008;68:5812-9. https://doi.org/10.1158/ 0008-5472.CAN-07-6387.

18. Gaustad JV, Brurberg KG, Simonsen TG, Mollatt CS, Rofstad EK. Tumor vascularity assessed by magnetic resonance imaging and intravital microscopy imaging. Neoplasia. 2008;10:354-62. https://doi.org/10.1593/neo.08162.

19. Schafer R, Leung HM, Gmitro AF. Multi-modality imaging of a murine mammary window chamber for breast cancer research. Biotechniques. 2014;57:45-50. https://doi.org/10.2144/00011 4191.

20. Laschke MW, Vollmar B, Menger MD. The dorsal skinfold chamber: window into the dynamic interaction of biomaterials with their surrounding host tissue. Eur Cell Mater. 2011;22:147-64.

21. Gregory MP, Andrew NF, Siqing S, Gabi H, Guoqing Z, Cassandra LF, et al. In vivo optical molecular imaging and analysis in mice using dorsal window chamber models applied to hypoxia, vasculature and fluorescent reporters. Nat Protoc. 2011;6:135566. https://doi.org/10.1038/nprot.2011.349.

22. Wang Q, Tous J, Liu Z, Ziegler S, Shi K. Evaluation of Timepix silicon detector for the detection of $18 \mathrm{~F}$ positrons. J Instrument. 2014;9:C05067.

23. Wang Q, Liu Z, Ziegler SI, Shi K. Enhancing spatial resolution of (18)F positron imaging with the Timepix detector by classification of primary fired pixels using support vector machine. Phys Med Biol. 2015;60:5261-78. https://doi.org/10.1088/0031-9155/60/13/ 5261.

24. Romano A, Tavanti F, Rossi Espagnet MC, Terenzi V, Cassoni A, Suma G, et al. The role of time-resolved imaging of contrast kinetics (TRICKS) magnetic resonance angiography (MRA) in the evaluation of head-neck vascular anomalies: a preliminary experience. Dentomaxillofac Radiol. 2015;44:20140302. https:// doi.org/10.1259/dmfr.20140302.

25. Damon J. Properties of ridges and cores for two-dimensional images. J Math Imaging Vis. 1999;10:163-74. https://doi.org/10. 1023/A:1008379107611. 
26. Wehrl HF, Sauter AW, Divine MR, Pichler BJ. Combined PET/ MR: a technology becomes mature. J Nucl Med. 2015;56:165-8. https://doi.org/10.2967/jnumed.114.150318.

27. Hundshammer C, Braeuer M, Muller CA, Hansen AE, Schillmaier M, Duwel S, et al. Simultaneous characterization of tumor cellularity and the Warburg effect with PET, MRI and hyperpolarized (13)C-MRSI. Theranostics. 2018;8:4765-80. https://doi.org/10. 7150/thno.25162.

28. Fanti S, Minozzi S, Antoch G, Banks I, Briganti A, Carrio I, et al. Consensus on molecular imaging and theranostics in prostate cancer. Lancet Oncol. 2018;19:e696-708. https://doi.org/10.1016/ S1470-2045(18)30604-1.

29. Gennaro N, Marrari A, Renne SL, Cananzi FCM, Quagliuolo VL, Di Brina L, et al. Multimodality imaging of adult rhabdomyosarcoma: the added value of hybrid imaging. Br J Radiol. 2020;93:20200250. https://doi.org/10.1259/bjr.20200250.

30. Catalano OA, Horn GL, Signore A, Iannace C, Lepore M, Vangel $\mathrm{M}$, et al. PET/MR in invasive ductal breast cancer: correlation between imaging markers and histological phenotype. Br J Cancer. 2017;116:893-902. https://doi.org/10.1038/bjc.2017.26.

31. Preibisch C, Shi K, Kluge A, Lukas M, Wiestler B, Gottler J, et al. Characterizing hypoxia in human glioma: a simultaneous multimodal MRI and PET study. NMR in biomedicine. 2017;30. https://doi.org/10.1002/nbm.3775.

32. Berker Y, Li Y. Attenuation correction in emission tomography using the emission data-a review. Med Phys. 2016;43:807-32. https://doi.org/10.1118/1.4938264.

33. Moses WW. Fundamental limits of spatial resolution in PET. Nucl Instrum Methods Phys Res A. 2011;648:S236-40. https://doi.org/ 10.1016/j.nima.2010.11.092.

34. Liu Z, Zhang P, Ji H, Long Y, Jing B, Wan L, et al. A mini-panel PET scanner-based microfluidic radiobioassay system allowing high-throughput imaging of real-time cellular pharmacokinetics. Lab Chip. 2020;20:1110-23. https://doi.org/10.1039/C9LC0 $1066 \mathrm{~A}$

35. Stickel JR, Cherry SR. High-resolution PET detector design: modelling components of intrinsic spatial resolution. Phys Med Biol. 2005;50:179-95

36. Liu Z, Jian Z, Wang Q, Cheng T, Feuerecker B, Schwaiger M, et al. A continuously infused microfluidic radioassay system for the characterization of cellular pharmacokinetics. J Nucl Med. 2016;57:1548-55. https://doi.org/10.2967/jnumed.115.169151.

37. Hofheinz F, Langner J, Petr J, Beuthien-Baumann B, Oehme L, Steinbach J, et al. A method for model-free partial volume correction in oncological PET. EJNMMI Res. 2012;2:16. https://doi. org/10.1186/2191-219X-2-16.

38. Cysouw MCF, Golla SVS, Frings V, Smit EF, Hoekstra OS, Kramer GM, et al. Partial-volume correction in dynamic PETCT: effect on tumor kinetic parameter estimation and validation of simplified metrics. EJNMMI Res. 2019;9:12. https://doi.org/ 10.1186/s13550-019-0483-z.

39. Sadeghi MM. (18)F-FDG PET and vascular inflammation: time to refine the paradigm? J Nucl Cardiol. 2015;22:319-24. https:// doi.org/10.1007/s12350-014-9917-1.

40. Kim M, Achmad A, Higuchi T, Arisaka Y, Yokoo H, Yokoo S, et al. Effects of intratumoral inflammatory process on 18F-FDG uptake: pathologic and comparative study with $18 \mathrm{~F}-$ fluoro- $\alpha-$ methyltyrosine PET/CT in oral squamous cell carcinoma. J Nucl Med. 2015;56:16-21. https://doi.org/10.2967/jnumed.114. 144014.

Publisher's note Springer Nature remains neutral with regard to jurisdictional claims in published maps and institutional affiliations. 